Original article

\title{
Smokeless tobacco quitting during COVID-19: A mixed-methods pilot study among participants screened for a cessation trial in India
}

\author{
Prashant Kumar Singh ${ }^{\text {a,b, }}$, Pankhuri Jain ${ }^{\mathrm{b}}$, Varsha Pandey ${ }^{\mathrm{b}}$, Shikha Saxena ${ }^{\mathrm{b}}$, Surbhi Tripathi ${ }^{\mathrm{b}}$, \\ Anuj Kumar ${ }^{c}$, Lucky Singh ${ }^{\mathrm{d}}$, Shalini Singh ${ }^{\mathrm{a}, \mathrm{b}}$ \\ ${ }^{a}$ WHO-FCTC Global Knowledge Hub on Smokeless Tobacco, ICMR National Institute of Cancer Prevention and Research, Noida 201301 Uttar Pradesh, India \\ ${ }^{\mathrm{b}}$ Division of Preventive Oncology \& Population Health, ICMR National Institute of Cancer Prevention and Research, Noida 201301 Uttar Pradesh, India \\ ${ }^{c}$ Division of Molecular Biology, ICMR National Institute of Cancer Prevention and Research, Noida 201301 Uttar Pradesh, India \\ ${ }^{\mathrm{d}}$ ICMR National Institute of Medical Statistics, Ansari Nagar, Delhi, India
}

\section{A R T I C L E I N F O}

\section{Keywords:}

Smokeless tobacco

COVID-19

Quitting

India

\begin{abstract}
A B S T R A C T
Introduction: COVID-19 and subsequent country-wide lockdown has impacted smokeless tobacco (SLT) product availability in India. We aimed to examine SLT quitting during COVID-19 lockdown among SLT users who consented to be enrolled in a cessation programme.

Methods: Between January-March 2020, we screened 227 exclusive SLT users to be enrolled in a randomizedcontrolled feasibility study on SLT cessation. However, all activities were suspended due to national lockdown in response to the COVID-19 pandemic. To examine the quitting intention and behaviour during COVID-19 lockdown, we re-contacted these individuals telephonically; during September-October 2020.

Results: Of 227 participants, 87 (38.3\%) could not be contacted on phone. We conducted telephonic qualitative interviews and assessed the SLT use status, willingness to quit and participate in the SLT cessation trial among the remaining 140 participants. Among these, $12.1 \%(17 / 140)$ showed no willingness to participate in the study due to migration. Since COVID-19 lockdown, 32.1\% (45/140) participants reported quitting SLT due to nonavailability, increased cost of products, shifts in community norms and family pressures.

Conclusions: COVID-19 pandemic presented an opportunity for tobacco cessation as stringent bans and isolation from social circles enabled tobacco cessation. It also triggered improvement in dissemination of public health information at an unprecedented scale, particularly related to the vulnerability of tobacco users to co-morbidities and harm from SARS CoV-2 infection. Implementation of strict bans on sale and consumption of SLT and strengthening of cessation support may lead to sustainable tobacco control. This study provides insight into effective policy strategies to reduce SLT use; which need to be substantiated with adequate cessation support.
\end{abstract}

\section{Introduction}

Smokeless tobacco (SLT) contains highly addictive and carcinogenic substances. ${ }^{1}$ Owing to its social acceptability and cultural sanction, SLT is used by over 300 million people, primarily in South Asian nations. ${ }^{2,3}$ Globally, 2.5 million Disability Adjusted Life Years (DALYs) and over 90, 000 lives were lost in 2017 due to diseases attributable to SLT use. ${ }^{4}$

Since the outbreak of COVID-19, concerns have emerged that individuals with existing co-morbidities may yield poorer clinical outcomes. ${ }^{5,6}$ Tobacco has been identified as a factor leading to weakening of the immune system and a dysfunctional immune response against SARS-CoV-2 may lead to greater viral dissemination. ${ }^{7}$ SLT use is of particular concern owing to the heavy burden of use in South Asian countries with high population densities, and increased susceptibility to co-morbidities. ${ }^{8,9}$ Research is warranted to understand the tobacco use patterns during COVID-19; especially in low-and-middle-income nations where majority of the tobacco users reside; specifically in South and Southeast Asia. ${ }^{4,10}$

\footnotetext{
* Corresponding author. WHO-FCTC Global Knowledge Hub on Smokeless Tobacco, ICMR National Institute of Cancer Prevention and Research, Noida 201301 Uttar Pradesh, India.

E-mail addresses: prashants.geo@gmail.com (P.K. Singh), pankhuri.pj@gmail.com (P. Jain), varsha.pandey.economics@gmail.com (V. Pandey), shikha.nicpr@


com (S. Singh).
} 
The modes of SLT preparation and consumption such as tobacco apparatus sharing; mashing the products in the palms; using fingers to place the product in the oral cavity and public spitting accompanying SLT use may increase the possibility of transmission from hand to mouth. ${ }^{11,12}$ Considering this, a few states in India have attempted to utilize the pandemic as an opportunity for strengthening tobacco control in the region through the prohibition of public spitting and mass appeals against tobacco use by eminent Indian personalities. ${ }^{13,14}$ Further, in some states and districts, the manufacture and sale of SLT products was also banned, and a uniform ban on all public use of SLT products, across all states has been mandated since April 2020. ${ }^{15}$ Studies suggest that COVID-19 presented an opportunity for tobacco cessation as legislative measures such as nationwide lockdown, ${ }^{16}$ closure of points of tobacco sale, stringent punishable offence, isolation from social circles and removal of certain cues may have enabled tobacco cessation among those already intending to quit and create intentions to quit among regular users.

Current evidence has been on smoking ${ }^{17}$ or e-cigarette use ${ }^{18,19}$ but research on SLT availability, quit rate and cessation during COVID-19 lockdown have not been reported widely. Further, current modes of SLT cessation include pharmacotherapy and counselling, requiring frequent visits to health centers which may have been hampered due to COVID-19 pandemic. $^{20}$ Gupte, et. al, 2020 have reported that the lockdown presented itself as an opportunity for quitting tobacco among those currently under treatment for tobacco cessation. ${ }^{21}$ This was particularly observed since the direct impact of the lockdown led to unavailability of tobacco products and increased prices due to closure of shops and vendors.

In this study, SLT quitting rate was assessed among the users who were included in a screening survey for SLT cessation trial during January-February 2020 (before the COVID-19 national lockdown i.e. March to June 2020) and reasons of quitting SLT during the follow-up in September-October 2020.

\section{Methods}

This section elucidates the selection of sample, recruitment and background of the study participants in addition to the tools utilised for conducting this study.

\subsection{Sample, recruitment and study setting}

Addressing Smokeless Tobacco use and building Research capacity in South Asia (ASTRA) is a randomized-controlled feasibility trial (RCT) to enable SLT cessation. Through interactive sessions and tobacco cessation counselling by experienced counsellors, the study aims to understand the efficacy of the intervention in enabling SLT cessation. As a part of this pilot study, undertaken for the RCT, we initiated participant screening in January 2020, ${ }^{22}$ however, due to the imposition of the national lockdown in India from March 2020, these activities were suspended. ${ }^{23}$ Based on the linkages of SLT use and COVID-19, we assessed the change in SLT use during the COVID-19 pandemic among participants screened for recruitment in the trial.

At ICMR-National Institute of Cancer Prevention and Research (ICMR-NICPR), Noida, Uttar Pradesh, India free and publicly-accessible oral, breast and cervical cancer screening is provided. Among those individuals undergoing screening for oral cancer, a screening module was administered to obtain information on their demographics, tobacco use patterns and intention to quit SLT in the next 30 days. Those individuals aged $\geq 18$ years and self-reported daily users of SLT for at least the last 6 months or using SLT for at least 25 days in the past one month were included in the study. We excluded individuals who self-reported use of combustible tobacco products in the past 30 days. ${ }^{24}$ Pregnant or breastfeeding women, cancer patients, and those with medical conditions that contra-indicate Nicotine Replacement Therapy (NRT) and those undergoing treatment for tobacco cessation were excluded.
Table 1

Demographic attributes of study participants at the first contact (January-March 2020) and second contact (September-October 2020) along with quitting rates.

\begin{tabular}{lll}
\hline January-February 2020 (Baseline) & $\mathrm{N}$ & $\%$ \\
\hline Total & 227 & $100.0 \%$ \\
Male & 184 & $81.1 \%$ \\
Female & 43 & $18.9 \%$ \\
$18-24$ & & \\
$25-29$ & 26 & $11.5 \%$ \\
$30-34$ & 39 & $17.2 \%$ \\
$35-39$ & 41 & $18.1 \%$ \\
$40-44$ & 38 & $16.7 \%$ \\
$45-49$ & 39 & $17.2 \%$ \\
$50+$ & 19 & $8.4 \%$ \\
\hline September 2020 (Re-contacted) & 25 & $11.0 \%$ \\
Call not reachable & - & \\
Phone switched-off & 20 & $8.8 \%$ \\
Call not answered & 38 & $16.7 \%$ \\
Call received & 29 & $12.8 \%$ \\
& 140 & $61.7 \%$ \\
\hline
\end{tabular}

$* x^{2}$ test was significant at $<0.05$

Written informed consent was obtained from the eligible participants after the objectives of the study and the expected outcomes were elucidated by the research team.

However, due to the imposition of the nation-wide lockdown, all activities barring essential services were suspended; hence, the RCT could not be delivered. At the beginning of the lockdown, 232 exclusive SLT users provided consent to participate in the study and were followed-up in September-October 2020.

During the stage of re-contacting, the interviews lasted $20-40 \mathrm{~min}$, and we collected information through in-depth interviews on tobacco consumption, including any use of combustible and/or SLT products (in the past seven days) whether the pandemic had affected their tobacco use, place of current residence, quitting of tobacco wherein abstinence was recorded for at least seven days, and willingness to quit tobacco/ participate in the clinical trial for SLT cessation. These calls were made by qualitative researchers and field investigators sensitized on tobacco use and from the background of public health.

The participants had consented for follow-up calls during the recruitment; however, verbal consent was obtained to conduct the telephonic interviews due to COVID-19 related precautionary guidelines. Further, all the participants in the follow-up calls were informed that their information may be used for research purposes without mentioning their personal identities including Name and Place. Throughout this study a team of researchers trained in qualitative data collection, tobacco cessation with a background of public health and dentistry were involved.

\subsection{Data analysis}

A descriptive analysis was carried out to report the change in status of SLT use among the participants. We estimated the proportion of participants that were successfully contacted by age and sex. Analyses were performed on STATA Version $14 .{ }^{25}$ Among those individuals who reported quitting SLT, we examined their reasons to quit.

\section{Results}

232 exclusive SLT users were screened between January-March 2020. Contact details were provided by 227 participants. None of the participants reported using combustible tobacco products. Table 1 provides the demographic details of the study participants by age and sex. Out of 227 participants, $61.7 \%$, i.e., 140 SLT users were successfully contacted through telephonic structured interview schedules. Interviews 




Fig. 1. Quitting rates of smokeless tobacco among study participants re-contacted during September-October, 2.

$x^{2}$ test was significant at $<0.05$

lasted for an average of $20 \mathrm{~min}$. Reasons for non-participation among the remaining $87(38.3 \%)$ participants included non-response to telephone call $(\mathrm{n}=29,33.3 \%)$, phone being switched-off $(\mathrm{n}=38,43.7 \%)$ and calls being not reachable $(\mathrm{n}=20,23.0 \%)$.

Of 140 participants, $45(32.1 \%)$ reported quitting SLT use completely wherein abstinence was reported in at least the past seven days. As observed in Fig. 1, the largest proportion of quitting was observed in those aged $\geq 50$ years, with a dip among those in middle adulthood between the ages 35-40 and 45-49 [Fig. 1]. About, 12.1\% (n $=17$ ) showed low/no willingness to participate in the clinical trial with a greater proportion of males at $13.7 \%(n=16)$ as compared to females $(4.4 \%, \mathrm{n}=1)$. Further, $9(6.4 \%)$ participants showed willingness to participate in the SLT cessation study; however, since they were not in the proximity of the study site and had migrated to their native places, it was not feasible for them to be included in the clinical trial.

Among participants who reported quitting, many quoted unavailability of SLT products in local markets to be a common reason. They substituted SLT products with traditional mouth fresheners and natural products like clove or fennel seeds.

"I used to carry pre-prepared pouches of fennel seeds and black pepper to the office to control cravings" (male, age 40).

Further, family pressures that may have arisen due to increase in dissemination of information of ill-effects of tobacco use during COVID19 pandemic, via news channels, social media, etc., may have led to cessation attempts. One participant who previously consumed 20 packs of SLT on an average day; said:

"My children sat with me and my wife. They all suggested me to quit for the sake of a healthy future. For few days, I felt as if I have lost a part of myself, Now, I have quit everything completely" (male, age 40).

The participants also reported that change in their social environment and public advisories against spitting have also enabled cessation. This change in community norm has prevented many users from using SLT in the desired form and motivated them further to quit.

"We have a social problem linked with my brother's habit of chewing tobacco. He is spitting in the proximity of the neighbourhood that is leading to quarrels with our peers. At the times of COVID-19, people are apprehensive if someone is spitting in their territory" (male, age 42).

\section{Discussion}

We found that one-third of the participants screened in
January-March 2020, that were followed-up, reported quitting SLT use, owing to non-availability of products, stringent bans on SLT sale, spitting ban in public places, and cessation advice based on changing community norms due to COVID-19 and subsequent lockdown. A significant proportion showed no willingness to participate in the clinical trial for SLT cessation due to migration consequent to COVID-19 lockdowns.

Our findings are consistent with studies undertaken to assess changes in tobacco use since the outbreak of COVID-19 as tobacco users report higher quitting intentions. ${ }^{26-29}$ To our knowledge, while there are studies assessing tobacco cessation patterns since the COVID-19 outbreak, our study is the only study showing quitting among those who first reported using SLT regularly before the pandemic and reasons for quitting since the outbreak without any medical intervention and based on circumstances presented by the pandemic. These insights have enabled us to come up with strategies that may be implemented that do not require cost-intensive medical interventions and are based on willingness to quit.

However, there are several limitations. First, we cannot verify the SLT use of those who could not be contacted. Second, we were only able to assess the SLT use at the time of contact and cannot determine longterm abstinence or relapse. Third, while we assessed reasons for quitting SLT, we were not able to ascertain the psychological status from 'forced' or circumstantial quitting. Fourth, we relied on self-reported change in SLT use and were not able to validate its veracity through biochemical assessment. Finally, since the study attempts to assess change in SLT use behaviour among those individuals who were already motivated to quit, the study findings are not generalizable and do not represent the average SLT user.

It is essential to examine the impact of COVID-19 lockdown policies especially related to tobacco cessation, specifically among different agegroups in future research, as we believe that an increase in quit intentions could be due to the improved dissemination of health promoting messages on news channels, social media and other platforms, especially on vulnerability of tobacco users to harm from COVID-19 and the risks posed by public spitting.

\section{Conclusions}

Our findings suggest that COVID-19, the subsequent lockdowns and legislative measures in response to public spitting, has presented an opportunity to enable SLT cessation among those users who reported to be motivated to quit, prior to the lockdown. The findings also show that unavailability of SLT products and the rising awareness of the ill-effects of SLT use on health among individuals in the social networks were 
factors that enabled effective cessation. Increased pricing of tobacco products and the decreased availability were observed to be important factors in increasing motivation to quit tobacco use in line with the WHO-MPOWER framework. ${ }^{30}$ Hence, we suggest an increase in regulation of point-of-sales of tobacco products and increase in pricing.

To translate quit attempts into sustainable and successful cessation, it is crucial to develop cessation support strategies in line with the emerging needs such as virtual support groups, and increased access to cessation support; especially among those who may have increased tobacco use due to psychological stress and anxiety. More research is needed to understand tobacco use patterns, specifically with SLT use, as these products are often not marketed commercially, can be locally produced, and may not be subject to changes in availability and pose challenge to the current cessation strategies. Also, it would be fruitful to explore the long-term abstinence rates of those users who have reported quitting during pandemic and offer cessation support as required. It would also be worthwhile to understand and explore tobacco use patterns during COVID-19 in other high-burden nations.

\section{Funding}

This research was funded by the National Institute for Health Research (NIHR) [ASTRA (Grant Reference Number 17/63/76)] using UK aid from the UK Government to support global health research. The views expressed in this publication are those of the author(s) and not necessarily those of the NIHR or the UK Department of Health and Social Care.'

\section{Ethical statement}

The study was reviewed and approved by the Institutional Review Board and the trial is registered at Clinical Trail Registry of India, Indian Council of Medical Research (CTRI number: CTRI/2019/01/016899).

\section{Declaration of competing interest}

The authors listed have no conflicts of interest to declare.

\section{References}

1 International Agency for Research on Cancer. Monographs on the Evaluation of Carcinogenic Risks to Humans - Volume 89. Smokeless Tobacco and Some Tobaccospecific N-Nitrosamines; 2007.

2 Kakde S, Bhopal RS, Jones CM. A Systematic Review on the Social Context of Smokeles Tobacco Use in the South Asian Population: Implications for Public Health. Public Health; 2012.

3 National Cancer Institute, Centers for Disease Control and Prevention. Smokeless Tobacco and Public Health: A Global Perspective. NIH Publication; 2014:14-7983.

4 Siddiqi K, Husain S, Vidyasagaran A, Readshaw A, Mishu MP, Sheikh A. Global burden of disease due to smokeless tobacco consumption in adults: an updated analysis of data from 127 countries. BMC Med. 2020 Aug 12;18(1):222.

5 Fang X, Li S, Yu H, et al. Epidemiological, comorbidity factors with severity and prognosis of COVID-19: a systematic review and meta-analysis. Aging. 2020.
6 Guan WJ, Liang WH, Zhao Y, et al. Comorbidity and its impact on 1,590 patients with Covid-19 in China: a nationwide analysis. Eur Respir J. 2020.

7 Gaunkar RB, Nagarsekar A, Carvalho KM, Jodalli PS, Mascarenhas K. COVID-19 in smokeless tobacco habitués: increased susceptibility and transmission. Cureus. 2020.

8 Boffetta P, Hecht S, Gray N, Gupta P, Straif K. Smokeless tobacco and cancer. Lancet Oncol. 2008.

9 Asthana S, Labani S, Kailash U, Sinha DN, Mehrotra R. Association of Smokeless Tobacco Use and Oral Cancer: A Systematic Global Review and Meta-Analysis. Nicotine $\&$ tobacco research : official journal of the Society for Research on Nicotine and Tobacco; 2019.

10 CDC Foundation, World Health Organization, Lung Foundation. The GATS Atlas Global Adult Tobacco Survey. 2015.

11 Kaur J, Rinkoo AV. Public Health Perspectives of Smokeless Tobacco and Areca Nut Use in the COVID-19 Era. Nicotine \& tobacco research : official journal of the Society for Research on Nicotine and Tobacco; 2020.

12 Gaunkar RB, Nagarsekar A, Carvalho KM, Jodalli PS, Mascarenhas K. COVID-19 in smokeless tobacco habitués: increased susceptibility and transmission. Cureus. 2020. 13 Ministry of Home Affairs. Order. 2020.

14 The Indian Express. Don't Consume or Spit Tobacco Products: ICMR amid Coronavirus Outbreak- the New Indian Express. 2020.

15 Vidyasagaran A, Arora M, Shukla R, Kidwai Z. Policy Responses to Smokeless Tobacco (ST) in India during the COVID19 Pandemic. ORF; 2020.

16 The Lancet. India under COVID-19 lockdown. The Lancet. 2020.

17 Kowitt SD, Ross JC, Jarman KL, et al. Tobacco quit intentions and behaviors among cigar smokers in the United States in response to covid-19. Int J Environ Res Publ Health. 2020.

18 Klemperer EM, West JC, Peasley-Miklus C, Villanti AC. Change in Tobacco and Electronic Cigarette Use and Motivation to Quit in Response to COVID-19. Nicotine \& Tobacco Research. official journal of the Society for Research on Nicotine and Tobacco; 2020.

19 Yach D. Tobacco Use Patterns in Five Countries during the COVID-19 Lockdown. Nicotine \& tobacco research : official journal of the Society for Research on Nicotine and Tobacco; 2020

20 Ruhil R. Correlates of the Use of Different Tobacco Cessation Methods by Smokers and Smokeless Tobacco Users According to Their Socio-Demographic Characteristics: Global Adult Tobacco Survey (GATS) India 2009-10. In: Indian Journal of Community Medicine. vol. 41. Medknow Publications; 2016:190-197.

21 Gupte H, Mandal G, Jagiasi D. How Has the COVID-19 Pandemic Affected Tobacco Users in India: Lessons from an Ongoing Tobacco Cessation Program. Tobacco Prevention \& Cessation; 2020.

22 Readshaw A, Mehrotra R, Mishu M, et al. Addressing smokeless tobacco use and building research capacity in South Asia (ASTRA). Journal of Global Health. 2020.

23 The Lancet. India under COVID-19 lockdown. The Lancet. 2020.

24 Readshaw A, Mehrotra R, Mishu M, et al. Addressing smokeless tobacco use and building research capacity in South Asia (ASTRA). Journal of Global Health. 2020.

25 StataCorp. Stata. Release 14. College Station, TX: Statistical Software; 2015. StataCorp LP.

26 Siddiqi K, Siddiqui F, Khan A, et al. The Impact of COVID-19 on Smoking Patterns in Pakistan: Findings from a Longitudinal Survey of Smokers. Nicotine \& Tobacco Research; 2020.

27 Kowitt SD, Ross JC, Jarman KL, et al. Tobacco quit intentions and behaviors among cigar smokers in the United States in response to covid-19. Int J Environ Res Publ Health. 2020.

28 Klemperer EM, West JC, Peasley-Miklus C, Villanti AC. Change in Tobacco and Electronic Cigarette Use and Motivation to Quit in Response to COVID-19. Nicotine \& Tobacco Research. official journal of the Society for Research on Nicotine and Tobacco; 2020.

29 Gupte H, Mandal G, Jagiasi D. How Has the COVID-19 Pandemic Affected Tobacco Users in India: Lessons from an Ongoing Tobacco Cessation Program. Tobacco Prevention \& Cessation; 2020.

30 Saxena A, Sharma K, Avashia V. Assessment of tobacco control policy in India: an evaluation using the World Health Organization MPOWER framework. Indian $J$ Community Med. 2020;45(4):542. 